\title{
Feasibility of set-valued implicit complementarity problems
}

Ren-you Zhong, Xiao-guo Wang and Jiang-hua Fan*

\section{"Correspondence:}

jhfan@mailbox.gxnu.edu.cn Department of Mathematics, Guangxi Normal University, Guilin, Guangxi, 541004, P.R. China

\begin{abstract}
In this paper, we introduce new concepts of $(\alpha, \beta, \gamma)$-exceptional family of elements and $(\alpha, \gamma)$-exceptional family of elements for the set-valued implicit complementarity problems in $R^{n}$ and infinite-dimensional Hilbert spaces, respectively. By utilizing these notions and the Leray-Schauder type fixed point theorem, we study the feasibility and strict feasibility of the set-valued implicit complementarity problems. Our results generalize some corresponding previously known results in the literature.
\end{abstract}

MSC: 49J40; $90 C 31$

Keywords: exceptional family of elements; set-valued implicit complementarity problems; Leray-Schauder type fixed point theorem; feasibility; strict feasibility

\section{Introduction}

Let $(H,\langle\cdot, \cdot\rangle)$ be a Hilbert space, $f, g: H \rightarrow 2^{H}$ be two set-valued mappings and $K$ be a cone with its dual cone $K^{*}$. The set-valued implicit complementarity problem defined by the (ordered) pair of mappings $(f, g)$ and $K$ is

$$
\operatorname{SICP}(f, g, K):\left\{\begin{array}{l}
\text { find } x^{*} \in H \text { such that there exist } \\
u^{*} \in f\left(x^{*}\right) \cap K^{*} \text { and } v^{*} \in g\left(x^{*}\right) \cap K \text { satisfying } \\
\left\langle u^{*}, v^{*}\right\rangle=0 .
\end{array}\right.
$$

If $f, g$ are single-valued mappings, $\operatorname{SICP}(f, g, K)$ reduces to the implicit complementarity problem

$$
\operatorname{ICP}(f, g, K):\left\{\begin{array}{l}
\text { find } x^{*} \in H \text { such that } \\
f\left(x^{*}\right) \in K^{*}, g\left(x^{*}\right) \in K \text { and }\left\langle f\left(x^{*}\right), g\left(x^{*}\right)\right\rangle=0 .
\end{array}\right.
$$

If $g=I$ (the identity mapping), $\operatorname{SICP}(f, g, K)$ reduces to the complementarity problem

$$
\mathrm{CP}(f, K):\left\{\begin{array}{l}
\text { find } x^{*} \in K \text { such that there exists } \\
u^{*} \in f\left(x^{*}\right) \cap K^{*} \text { satisfying }\left\langle u^{*}, x^{*}\right\rangle=0 .
\end{array}\right.
$$

$\operatorname{SICP}(f, g, K)$ is said to be feasible if

$$
\left\{x \in H: f(x) \cap K^{*} \neq \emptyset, g(x) \cap K \neq \emptyset\right\} \neq \emptyset ;
$$

\section{Springer}

(0) 2013 Zhong et al:; licensee Springer. This is an Open Access article distributed under the terms of the Creative Commons Attribution License (http://creativecommons.org/licenses/by/2.0), which permits unrestricted use, distribution, and reproduction in any medium, provided the original work is properly cited. 
$\operatorname{SICP}(f, g, K)$ is said to be strictly feasible if

$$
\left\{x \in H: f(x) \cap \operatorname{int} K^{*} \neq \emptyset, g(x) \cap K \neq \emptyset\right\} \neq \emptyset .
$$

Complementarity theory has been intensively considered due to its various applications in operations research, economic equilibrium and engineering design. The reader is referred to $[1,2]$ and the reference therein. The implicit complementarity problem was introduced into the complementarity theory in [3] as a mathematical tool in the study of some stochastic optimal control problems.

Strict feasibility plays an important role in the development of the theory and algorithms of complementarity problems. It is closely related to the solvability of the complementarity problems. For example, when $f$ is a quasi(pseudo)monotone map, or more generally, a quasi- $P_{*}$-map, then the strict feasibility is sufficient for the solvability of the $\mathrm{CP}$; for more details, see [4-7]. An important method in studying the feasibility of the complementarity problems is based on the concept of an exceptional family of elements for a continuous function. In recent years, several authors have been dedicated to the feasibility of the (implicit) complementarity problems by using the exceptional family of elements method; for example, see [8-11].

At the end of the paper [9], Isac proposed three open problems and two of them can be extracted as follows.

$\left(\mathrm{Q}_{1}\right)$ Are Theorem 5.2 and Theorem 6.1 true without the assumption $K^{*} \subseteq K$ ?

$\left(\mathrm{Q}_{2}\right)$ Can the method presented in this paper be adapted to the study of strict feasibility?

Huang et al. [8] and Yoel et al. [11] considered the solvability of problems $\left(\mathrm{Q}_{1}\right)$ and $\left(\mathrm{Q}_{2}\right)$, respectively. In [8], they introduced new concepts of $\alpha$-exceptional family of elements and $(\alpha, \beta)$-exceptional family of elements for continuous functions and studied the feasibility for nonlinear complementarity problems in $R^{n}$ and an infinite-dimensional Hilbert space $H$ without the assumption $K^{*} \subseteq K$. In [11], based on their new concepts of $(\alpha, \gamma)$ exceptional family of elements and $(\alpha, \beta, \gamma)$-exceptional family of elements and the topological degree theory, they studied the feasibility and strict feasibility of $\operatorname{ICP}(f, g, K)$ in $R^{n}$ and an infinite-dimensional Hilbert space $H$, which partly answered the open problem $\left(\mathrm{Q}_{2}\right)$.

Isac et al. [10] introduced a new notion of exceptional family of elements for the pair of $(f, g)$ involved in the implicit complementarity problems. By employing the LeraySchauder alternative, they gave more general existence theorems for $\operatorname{ICP}(f, g, K)$ and $\operatorname{SICP}(f, g, K)$ when $f, g$ are set-valued lower semicontinuous mappings with closed convex values. When $f$ is a set-valued upper semicontinuous mapping with closed convex values, $g$ is a one-to-one mapping, [10] established some new existence theorems.

Many of the well-known existence theorems for the problem $\operatorname{ICP}(f, g, K)$ demand that the mappings $f$ and $g$ be subject to some strong restrictions. For example, Isac [12, 13] required that $f$ be strongly monotone and Lipschitz continuous with respect to $g$.

Motivated by the works mentioned above, we introduce new concepts of $(\alpha, \beta, \gamma)$ exceptional family of elements and $(\alpha, \gamma)$-exceptional family of elements for $\operatorname{SICP}(f, g, K)$ under weaker restrictions on the mappings $f$ and $g$. By utilizing these notions and the Leray-Schauder type fixed point theorem proposed in [14], we investigate the (strict) feasibility of $\operatorname{SICP}(f, g, K)$ in $R^{n}$ and infinite-dimensional Hilbert spaces, respectively. The 
results presented in this paper not only answer the above open problems $\left(\mathrm{Q}_{1}\right)$ and $\left(\mathrm{Q}_{2}\right)$ proposed in [9], but also generalize some corresponding previously known results in $[8$, $9,11,15]$.

The paper is arranged in the following way. In Section 2, we recall some required concepts and basic results for the later use. In Section 3, we introduce new concepts of $(\alpha, \beta, \gamma)$-exceptional family of elements and $(\alpha, \gamma)$-exceptional family of elements for $\operatorname{SICP}(f, g, K)$ and discuss the (strict) feasibility in $R^{n}$. In Section 4 , by using the new notion of $(\alpha, \beta, \gamma)$-exceptional family of elements, we consider the (strict) feasibility for $\operatorname{SICP}(f, g, K)$ in infinite-dimensional Hilbert spaces.

\section{Notations and fundamental results}

Let $X$ and $Y$ be topological spaces, the collection of all nonempty compact subsets of $X$ is denoted by $c(X)$. For any subset $A$ of $X$, the interior, closure and boundary of $A$ are denoted by int $A, \bar{A}$ and $\partial A$, respectively. The relative boundary of $U$ in $K$ is denoted by $\partial_{K} U$.

Definition 2.1 The set-valued mapping $F: X \rightarrow 2^{Y}$ is said to be upper semicontinuous on $X$ if $\{x \in X: f(x) \subset V\}$ is open in $X$ whenever $V$ is an open subset of $Y$.

Definition 2.2 The set-valued mapping $F: X \rightarrow 2^{Y}$ is said to be compact if $F(X)$ is relatively compact in $Y$.

Definition 2.3 An upper semicontinuous set-valued mapping $F: X \rightarrow c(Y)$ is said to be admissible if there exist a topological space $Z$ and continuous functions $p: Z \rightarrow X$ and $q: Z \rightarrow Y$ satisfying

(1) $\emptyset \neq q\left(p^{-1} x\right) \subset F(x)$ for each $x \in X$;

(2) $p$ is proper; that is, the inverse image $p^{-1}(A)$ of any compact set $A \subset X$ is compact;

(3) for each $x \in X, p^{-1}(x)$ is an acyclic subset of $Z$.

A nonempty topological space $X$ is said to be acyclic provided that all of its reduced Cech homology groups over rational vanish. For a nonempty subset in a topological vector space, we have the following implications:

$$
\text { convex } \Longrightarrow \text { star-shaped } \Longrightarrow \text { contractible } \Longrightarrow \text { acyclic } \Longrightarrow \text { connected, }
$$

but not conversely.

It is well known that any upper semicontinuous set-valued mapping with compact acyclic values is admissible, and the composition of two admissible mappings is also admissible; see [14]. The admissible mapping is a large class of set-valued mappings, such a class contains composites of a lot of well-known set-valued mappings, which appear in nonlinear analysis and algebraic topology; for more details, see [16].

The following property of admissible maps can be found in [17].

Lemma 2.1 Let $X, Y$ be two topological spaces, E be a topological vector space, $F, G: X \rightarrow$ $c(E)$ be admissible, and let $f: Y \rightarrow R$ be continuous. Then the mappings

$$
\begin{aligned}
& S: X \rightarrow c(E), \quad S(x):=F(x)+G(x) \quad \text { for } x \in X, \\
& T: X \times Y \rightarrow c(E), \quad T(x, y):=f(y) F(x) \quad \text { for }(x, y) \in X \times Y
\end{aligned}
$$

are admissible. 
Let $E$ be a Banach space and $A \subset E$. The Kuratowski measure of noncompactness of $A$ is defined by

$$
\alpha(A)=\inf \left\{\epsilon>0: A \subset \bigcup_{i=1}^{n} A_{i} \text { and } \operatorname{diam}\left(A_{i}\right) \leq \epsilon \text { for } i=1,2, \ldots, n\right\},
$$

where $\operatorname{diam}(A)=\sup \{\|x-y\|: x, y \in A\}$. It is known that $\alpha(A)=0$ if and only if $A$ is relatively compact.

An upper semi-continuous map $F: E \rightarrow 2^{E}$ is said to be condensing if for any subset $B \subset E$ with $\alpha(B) \neq 0$, we have $\alpha(F(B))<\alpha(B)$.

Let $H$ be a Hilbert space, $K \subset H$ is a closed pointed convex cone if and only if $K$ is a closed subset of $H$ satisfying
(i) $K+K \subset K$;
(ii) $\lambda K \subset K, \forall \lambda \geq 0$;
(iii) $K \cap(-K)=\{0\}$.

The dual cone of $K$ is defined by

$$
K^{*}=\{y \in H:\langle y, x\rangle \geq 0, \forall x \in K\} .
$$

The following Leray-Schauder type fixed point theorem is a particular form of Corollary 4.2 in [17], which is the basis of our arguments in this paper.

Theorem 2.1 Let $H$ be a Hilbert space, $C \subset H$ be closed and convex and $U$ be a relatively open subset of $C$ with $0 \in U$. Suppose that $F: \bar{U} \rightarrow c(H)$ is a condensing admissible mapping such that

$$
F(x) \cap\{\lambda x: \lambda>1\}=\emptyset, \quad \forall x \in \partial_{K} U .
$$

Then $F$ has a fixed point in $\bar{U}$.

The projection operator onto $K$ is denoted by $P_{K}$, for every $x \in H, P_{K}(x)$ is the unique element in $K$ satisfying

$$
\left\|x-P_{K}(x)\right\|=\min _{y \in K}\|x-y\| .
$$

It is well known that, for each $x \in H$, the projection $P_{K}(x)$ of $x$ is characterized by the following properties:

$\left(\mathrm{P}_{1}\right)\left\langle P_{K}(x)-x, y\right\rangle \geq 0$ for all $y \in K$;

$\left(\mathrm{P}_{2}\right)\left\langle P_{K}(x)-x, P_{K}(x)\right\rangle=0$.

\section{Feasibility and strict feasibility in $R^{n}$}

In this section, we study the feasibility and strict feasibility of $\operatorname{SICP}(f, g, K)$ in $R^{n}$. We first introduce a new concept of $(\alpha, \beta, \gamma)$-exceptional family of elements (for short, $(\alpha, \beta, \gamma)$ EFE) for the pair $(f, g)$ with respect to $K$. 
Definition 3.1 Let $K$ be a closed pointed convex cone in $R^{n}$ with $K^{*} \subseteq K$ and int $K^{*} \neq \emptyset$. Let $\varepsilon: R^{n} \rightarrow c\left(\operatorname{int} K^{*}\right), f, g: R^{n} \rightarrow c\left(R^{n}\right)$ be admissible mappings. Given $\alpha, \beta, \gamma \geq 0$ with $0 \leq \alpha<\beta$, we say that the family of elements $\left\{x_{r}\right\}_{r>0} \subset R^{n}$ is an $(\alpha, \beta, \gamma)$-EFE for the pair $(f, g)$ with respect to $K$ if the following conditions are satisfied:

(1) $\left\|x_{r}\right\| \rightarrow+\infty$ as $r \rightarrow+\infty$;

(2) for any $r>0$, there exist $\mu_{r}>0$ and elements $f^{r} \in f\left(x_{r}\right), g^{r} \in g\left(x_{r}\right), \varepsilon^{r} \in \varepsilon\left(x_{r}\right)$ such that $s^{r}=\mu_{r} x_{r}+(\beta-\alpha) f^{r}-\gamma \varepsilon^{r} \in K^{*}, w^{r}=\mu_{r} x_{r}+g^{r}-\alpha f^{r} \in K$ and $\left\langle s_{r}, w_{r}\right\rangle=0$.

Remark 3.1 If $g=I, f$ is single-valued, $\gamma=0$ and $\mu_{r}=\frac{1}{t_{r}}-1$, then Definition 3.1 reduces to Definition 5.2 in [9].

Theorem 3.1 Let $K$ be a closed pointed convex cone in $R^{n}$ with $K^{*} \subseteq K$ and int $K^{*} \neq \emptyset$. Let $\varepsilon: R^{n} \rightarrow c\left(\right.$ int $\left.K^{*}\right), f, g: R^{n} \rightarrow c\left(R^{n}\right)$ be admissible mappings. Then either $\operatorname{SICP}(f, g, K)$ is feasible, or for any $\alpha, \beta, \gamma \geq 0$ with $0 \leq \alpha<\beta$, there exists an $(\alpha, \beta, \gamma)$-EFE (in the sense of Definition 3.1) for the pair $(f, g)$ with respect to $K$.

Moreover, if $\gamma>0$, then either $\operatorname{SICP}(f, g, K)$ is strictly feasible, or for any $\alpha \geq 0$, there exists an $(\alpha, \beta, \gamma)$-EFE (in the sense of Definition 3.1) for the pair $(f, g)$ with respect to $K$.

Proof Define $\phi: R^{n} \rightarrow c\left(R^{n}\right)$ by

$$
\phi(x)=g(x)-\alpha f(x)-P_{K}(g(x)-\beta f(x)+\gamma \varepsilon(x)), \quad \forall x \in H .
$$

Consider the equation

$$
0 \in \phi(x)
$$

We have the following two cases to discuss.

Case 1. If equation (3.1) has a solution in $R^{n}$, denoted by $x^{*}$, then there exist $u^{*} \in f\left(x^{*}\right)$, $v^{*} \in g\left(x^{*}\right)$ and $\epsilon^{*} \in \varepsilon\left(x^{*}\right)$ such that

$$
0=v^{*}-\alpha u^{*}-P_{K}\left(v^{*}-\beta u^{*}+\gamma \epsilon^{*}\right)
$$

that is,

$$
v^{*}-\alpha u^{*}=P_{K}\left(v^{*}-\beta u^{*}+\gamma \epsilon^{*}\right) .
$$

By the property $\left(\mathrm{P}_{1}\right)$ of $P_{K}$, we have

$$
\left\langle v^{*}-\alpha u^{*}-\left(v^{*}-\beta u^{*}+\gamma \epsilon^{*}\right), y\right\rangle \geq 0, \quad \forall y \in K,
$$

that is,

$$
\left\langle(\beta-\alpha) u^{*}-\gamma \epsilon^{*}, y\right\rangle \geq 0, \quad \forall y \in K .
$$

Then it follows from (3.3) that $(\beta-\alpha) u^{*}-\gamma \epsilon^{*} \in K^{*}$. Since $\gamma \geq 0$, it is clear that $u^{*} \in K^{*}$ and so $u^{*} \in f\left(x^{*}\right) \cap K^{*}$. From (3.2) we obtain that $v^{*} \in K+\alpha u^{*} \subset K+K^{*}$, since $K^{*} \subseteq K$, thus $v^{*} \in g\left(x^{*}\right) \cap K$. Then $\operatorname{SICP}(f, g, K)$ is feasible. 
Case 2. If equation (3.1) does not have a solution, set $\psi=I-\phi$, then the mapping $\psi$ has no fixed point in $R^{n}$. Thus, for any $r>0$, let $U_{r}=\left\{x \in R^{n}:\|x\|<r\right\}$, the mapping $\psi$ is fixed-point free with respect to the set $\overline{U_{r}}$.

By the continuity of $P_{K}$, the upper semicontinuity of $f, g$ and $\varepsilon$, it is clear to see that $\psi$ is upper semicontinuous. Since any upper semicontinuous mapping with compact values is compact in $R^{n}$, thus $\psi$ is compact.

Since $I, P_{K}, f, g$ and $\varepsilon$ all are admissible, it follows from Lemma 2.1 that $\psi$ is admissible.

Applying Theorem 2.1 with the mapping $\psi$ and the set $U=U_{r}$, there exist $x_{r} \in \partial U_{r}=$ $\{x \in H:\|x\|=r\}$ and $\lambda_{r}>1$ such that

$$
\lambda_{r} x_{r} \in x_{r}-\phi\left(x_{r}\right)
$$

Setting $\mu_{r}=\lambda_{r}-1$, we have

$$
\mu_{r} x_{r} \in-\phi\left(x_{r}\right)
$$

From (3.4), it can be deduced that there exist $f^{r} \in f\left(x_{r}\right), g^{r} \in g\left(x_{r}\right)$ and $\varepsilon^{r} \in \varepsilon\left(x_{r}\right)$ such that

$$
\mu_{r} x_{r}+g^{r}-\alpha f^{r}=P_{K}\left(g^{r}-\beta f^{r}+\gamma \varepsilon^{r}\right)
$$

Then the properties $\left(\mathrm{P}_{1}\right)$ and $\left(\mathrm{P}_{2}\right)$ of $P_{K}$ and (3.5) jointly yield that

$$
\left\langle\mu_{r} x_{r}+g^{r}-\alpha f^{r}-\left(g^{r}-\beta f^{r}+\gamma \varepsilon^{r}\right), y\right\rangle \geq 0, \quad \forall y \in K
$$

and

$$
\left\langle\mu_{r} x_{r}+g^{r}-\alpha f^{r}-\left(g^{r}-\beta f^{r}+\gamma \varepsilon^{r}\right), \mu_{r} x_{r}+g^{r}-\alpha f^{r}\right\rangle=0 .
$$

Or equivalently,

$$
\left\langle\mu_{r} x_{r}+(\beta-\alpha) f^{r}-\gamma \varepsilon^{r}, y\right\rangle \geq 0, \quad \forall y \in K
$$

and

$$
\left\langle\mu_{r} x_{r}+(\beta-\alpha) f^{r}-\gamma \varepsilon^{r}, \mu_{r} x_{r}+g^{r}-\alpha f^{r}\right\rangle=0 .
$$

Letting $s^{r}=\mu_{r} x_{r}+(\beta-\alpha) f^{r}-\gamma \varepsilon^{r}, w^{r}=\mu_{r} x_{r}+g^{r}-\alpha f^{r}$, then from (3.5) and (3.6), we obtain that $w^{r} \in K$ and $s^{r} \in K^{*}$. Thus, (3.7) implies that $\left\langle s^{r}, w^{r}\right\rangle=0$.

Since $x_{r} \in \partial U_{r}=\{x \in H:\|x\|=r\}$, we have $\left\|x_{r}\right\| \rightarrow+\infty$ as $r \rightarrow+\infty$.

Then $\left\{x_{r}\right\}_{r>0}$ is an $(\alpha, \beta, \gamma)$-EFE for the pair $(f, g)$ with respect to $K$, thus the first assertion of the theorem is proved.

If $\gamma>0$, then it follows from (3.3) that $(\beta-\gamma) u^{*}-\gamma \epsilon^{*} \in K^{*}$, thus $(\beta-\gamma) u^{*} \in K^{*}+\gamma \epsilon^{*} \subset$ int $K^{*}$. This finishes the proof of the second assertion of the theorem, which completes the proof as a whole. 
Remark 3.2 Since Definition 3.1 is a generalization of Definition 5.2 in [9], then Theorem 5.2 in [9] is a special case of Theorem 3.1.

We now introduce a new notion of $(\alpha, \gamma)$-exceptional family of elements (for short, $(\alpha, \gamma)$-EFE) for the pair $(f, g)$ with respect to $K$.

Definition 3.2 Let $K$ be a closed pointed convex cone in $R^{n}$ with int $K^{*} \neq \emptyset$. Let $\varepsilon: R^{n} \rightarrow$ $c\left(\right.$ int $\left.K^{*}\right), f, g: R^{n} \rightarrow c\left(R^{n}\right)$ be admissible mappings. Given $\alpha, \gamma \geq 0$, we say that the family of elements $\left\{x_{r}\right\}_{r>0} \subset R^{n}$ is an $(\alpha, \gamma)$-EFE for the pair $(f, g)$ with respect to $K$ if the following conditions are satisfied:

(1) $\left\|x_{r}\right\| \rightarrow+\infty$ as $r \rightarrow+\infty$;

(2) for any $r>0$, there exist $\mu_{r}>0$ and elements $f^{r} \in f\left(x_{r}\right), g^{r} \in g\left(x_{r}\right), \varepsilon^{r} \in \varepsilon\left(x_{r}\right)$ such that $s^{r}=\mu_{r} x_{r}+f^{r}-\gamma \varepsilon^{r} \in K^{*}, w^{r}=\mu_{r} x_{r}+g^{r}-\alpha P_{K}\left(f^{r}\right) \in K$ and $\left\langle s_{r}, w_{r}\right\rangle=0$.

\section{Remark 3.3}

(1) If $g=I$ and $f$ is single-valued, Definition 3.2 reduces to Definition 3.1 in [11];

(2) If $g=I, f$ is single-valued and $\gamma=0$, Definition 3.2 reduces to Definition 3.1 in [8];

(3) If $g=I, f$ is single-valued and $\alpha=\gamma=0$, Definition 3.2 reduces to Definition 5.1 in [9] or Definition 3 in [15].

The following theorem shows us that the conclusion is true without the assumption $K^{*} \subseteq K$, which answers the open problem in [9]. And the assumption on $f$ and $g$ is weaker than that in [11].

Theorem 3.2 Let $K$ be a closed pointed convex cone in $R^{n}$ with int $K^{*} \neq \emptyset$. Let $\varepsilon: R^{n} \rightarrow$ $c\left(\right.$ int $\left.K^{*}\right), f, g: R^{n} \rightarrow c\left(R^{n}\right)$ be admissible mappings. Then either $\operatorname{SICP}(f, g, K)$ is feasible, or for any $\alpha, \gamma \geq 0$, there exists an $(\alpha, \gamma)$-EFE (in the sense of Definition 3.2) for the pair $(f, g)$ with respect to $K$.

Moreover, if $\gamma>0$, then either $\operatorname{SICP}(f, g, K)$ is strictly feasible, or for any $\alpha \geq 0$, there exists an $(\alpha, \gamma)$-EFE (in the sense of Definition 3.2) for the pair $(f, g)$ with respect to $K$.

Proof Define $\phi: R^{n} \rightarrow c\left(R^{n}\right)$ by

$$
\phi(x)=g(x)-\alpha P_{K}(f(x))-P_{K}\left(g(x)-f(x)+\gamma \varepsilon(x)-\alpha P_{K}(f(x))\right), \quad \forall x \in H .
$$

Consider the equation

$$
0 \in \phi(x)
$$

We have the following two cases.

Case 1. If equation (3.8) has a solution in $R^{n}$, denoted by $x^{*} \in R^{n}$, then there exist $u^{*} \in$ $f\left(x^{*}\right), v^{*} \in g\left(x^{*}\right)$ and $\epsilon^{*} \in \varepsilon\left(x^{*}\right)$ such that

$$
0=v^{*}-\alpha P_{K}\left(u^{*}\right)-P_{K}\left(v^{*}-u^{*}+\gamma \epsilon^{*}-\alpha P_{K}\left(u^{*}\right)\right),
$$


that is,

$$
v^{*}-\alpha P_{K}\left(u^{*}\right)=P_{K}\left(v^{*}-u^{*}+\gamma \epsilon^{*}-\alpha P_{K}\left(u^{*}\right)\right) .
$$

It follows from (3.9) that $v^{*} \in K$ and thus $v^{*} \in g\left(x^{*}\right) \cap K$.

By the property $\left(\mathrm{P}_{1}\right)$ of $P_{K}$, we have

$$
\left\langle v^{*}-\alpha P_{K}\left(u^{*}\right)-\left(v^{*}-u^{*}+\gamma \epsilon^{*}-\alpha P_{K}\left(u^{*}\right)\right), y\right\rangle \geq 0, \quad \forall y \in K,
$$

which is

$$
\left\langle u^{*}-\gamma \epsilon^{*}, y\right\rangle \geq 0, \quad \forall y \in K
$$

It follows from (3.10) that $u^{*}-\gamma \epsilon^{*} \in K^{*}$. Since $\gamma \geq 0$, it is clear that $u^{*} \in K^{*}$. Then $u^{*} \in$ $f\left(x^{*}\right) \cap K^{*}$. Hence the problem $\operatorname{SICP}(f, g, K)$ is feasible.

Case 2. If equation (3.8) does not have a solution, set $\psi=I-\phi$, then the mapping $\psi$ has no fixed point in $R^{n}$. For any $r>0$, let $U_{r}=\left\{x \in R^{n}:\|x\|<r\right\}$, the mapping $\psi$ is fixed-point free with respect to the set $\overline{U_{r}}$.

By the continuity of the projection operator $P_{K}$, and the upper semicontinuity of $f, g$ and $\varepsilon$, it is clear to see that $\psi$ is upper semicontinuous. Since any upper semicontinuous mapping is compact in $R^{n}$, thus $\psi$ is compact.

Since $I, P_{K}, f, g$ and $\varepsilon$ all are admissible, it follows from Lemma 2.1 that $\psi$ is admissible.

Applying Theorem 2.1 with the mapping $\psi$ and the set $U=U_{r}$, we obtain that there exist $x_{r} \in \partial U_{r}=\{x \in H:\|x\|=r\}$ and $\lambda_{r}>1$ such that

$$
\lambda_{r} x_{r} \in x_{r}-\phi\left(x_{r}\right)
$$

Setting $\mu_{r}=\lambda_{r}-1$, we have

$$
\mu_{r} x_{r} \in-\phi\left(x_{r}\right)
$$

From (3.11), we deduce that there exist $f^{r} \in f\left(x_{r}\right), g^{r} \in g\left(x_{r}\right)$ and $\varepsilon^{r} \in \varepsilon\left(x_{r}\right)$ such that

$$
\mu_{r} x_{r}+g^{r}-\alpha P_{K}\left(f^{r}\right)=P_{K}\left(g^{r}-f^{r}+\gamma \varepsilon^{r}-\alpha P_{K}\left(f^{r}\right)\right) .
$$

From the properties $\left(\mathrm{P}_{1}\right)$ and $\left(\mathrm{P}_{2}\right)$ of $P_{K}$ and (3.12), we have

$$
\left\langle\mu_{r} x_{r}+g^{r}-\alpha P_{K}\left(f^{r}\right)-\left(g^{r}-f^{r}+\gamma \varepsilon^{r}-\alpha P_{K}\left(f^{r}\right)\right), y\right\rangle \geq 0, \quad \forall y \in K
$$

and

$$
\left\langle\mu_{r} x_{r}+g^{r}-\alpha P_{K}\left(f^{r}\right)-\left(g^{r}-f^{r}+\gamma \varepsilon^{r}-\alpha P_{K}\left(f^{r}\right)\right), \mu_{r} x_{r}+g^{r}\right\rangle=0 .
$$

Or equivalently,

$$
\left\langle\mu_{r} x_{r}+f^{r}-\gamma \varepsilon^{r}, y\right\rangle \geq 0, \quad \forall y \in K
$$


and

$$
\left\langle\mu_{r} x_{r}+f^{r}-\gamma \varepsilon^{r}, \mu_{r} x_{r}+g^{r}-\alpha P_{K}\left(f^{r}\right)\right\rangle=0 .
$$

Setting $s^{r}=\mu_{r} x_{r}+f^{r}-\gamma \varepsilon^{r}$ and $w^{r}=\mu_{r} x_{r}+g^{r}-\alpha P_{K}\left(f^{r}\right)$, from (3.12) and (3.13) we have that $w^{r} \in K$ and $s^{r} \in K^{*}$. Thus, (3.14) implies that $\left\langle s^{r}, w^{r}\right\rangle=0$.

Since $x_{r} \in \partial U_{r}=\{x \in H:\|x\|=r\}$, thus we have $\left\|x_{r}\right\| \rightarrow+\infty$ as $r \rightarrow+\infty$.

Then $\left\{x_{r}\right\}_{r>0}$ is an $(\alpha, \gamma)$-EFE for the pair $(f, g)$ with respect to $K$ and so the first assertion of the theorem is proved.

If $\gamma>0$, then it follows from (3.10) that $u^{*}-\gamma \epsilon^{*} \in K^{*}$ and so $u^{*} \in K^{*}+\gamma \epsilon^{*} \subset \operatorname{int} K^{*}$. This finishes the proof of the second assertion of the theorem, which completes the proof.

\section{Feasibility and strict feasibility in Hilbert spaces}

In this section, we study the feasibility and strict feasibility of the problem SICP in Hilbert spaces. The following $(\alpha, \beta, \gamma)$-EFE is new.

Definition 4.1 Let $H$ be a Hilbert space, $K \subset H$ be a closed convex cone with int $K^{*} \neq \emptyset$ and $K^{*} \subseteq K$. Let $\varepsilon: H \rightarrow c\left(\right.$ int $\left.K^{*}\right)$ be compact admissible mappings, and let $f, g: H \rightarrow$ $c(H)$ be admissible mappings such that $f(x)=\frac{1}{\beta} x-S(x), g(x)=x-T(x)$, where $\beta>0$, and $S, T: H \rightarrow c(H)$ are compact. Given $\alpha, \gamma \geq 0$ with $0 \leq \alpha<\beta$, we say that the family of elements $\left\{x_{r}\right\}_{r>0} \subset H$ is an $(\alpha, \beta, \gamma)$-EFE for the pair $(f, g)$ with respect to $K$, if the following conditions are satisfied:

(1) $\left\|x_{r}\right\| \rightarrow+\infty$ as $r \rightarrow+\infty$;

(2) for any $r>0$, there exist $\mu_{r}>0$ and elements $f^{r} \in f\left(x_{r}\right), g^{r} \in g\left(x_{r}\right)$ and $\varepsilon^{r} \in \varepsilon\left(x_{r}\right)$ such that $s^{r}=\frac{\mu_{r}}{\beta} x_{r}+f^{r}-\frac{1}{\beta-\alpha} \gamma \varepsilon^{r} \in K^{*}, w^{r}=(\beta-\alpha) \mu_{r} x_{r}+\beta g^{r}-\alpha \beta f^{r} \in K$ and $\left\langle s_{r}, w_{r}\right\rangle=0$.

Remark 4.1 If $g=I, f$ is single-valued, $\gamma=0$ and $\mu_{r}=\frac{1}{t_{r}}-1$, then Definition 4.1 reduces to Definition 6.1 in [9].

Theorem 4.1 Let $H$ be a Hilbert space, $K \subset H$ be a closed convex cone with int $K^{*} \neq \emptyset$ and $K^{*} \subseteq K$. Let $\varepsilon: H \rightarrow c\left(\operatorname{int} K^{*}\right)$ be a compact admissible mapping, and let $f, g: H \rightarrow c(H)$ be admissible mappings such that $f(x)=\frac{1}{\beta} x-S(x)$ and $g(x)=x-T(x)$, where $\beta>0$ and $S, T: H \rightarrow c(H)$ are compact. Then either $\operatorname{SICP}(f, g, K)$ is feasible, or for any $\gamma \geq 0$ and $\alpha \geq 0$ with $0 \leq \alpha<\beta$, there exists an $(\alpha, \beta, \gamma)$-EFE (in the sense of Definition 4.1) for the pair $(f, g)$ with respect to $K$.

Moreover, if $\gamma>0$, then either $\operatorname{SICP}(f, g, K)$ is strictly feasible, or for any $\alpha \geq 0$, there exists an $(\alpha, \beta, \gamma)$-EFE (in the sense of Definition 4.1) for the pair $(f, g)$ with respect to $K$.

Proof Define $\phi: H \rightarrow c(H)$ by

$$
\phi(x)=g(x)-\alpha f(x)-P_{K}(g(x)-\beta f(x)+\gamma \varepsilon(x)), \quad \forall x \in H .
$$

Consider the equation

$$
0 \in \phi(x)
$$

We consider the following two cases. 
Case 1. If the mapping $\phi$ has a zero point in $H$, denoted by $x^{*} \in H$, then there exist $u^{*} \in f\left(x^{*}\right), v^{*} \in g\left(x^{*}\right)$ and $\epsilon^{*} \in \varepsilon\left(x^{*}\right)$ such that

$$
0=v^{*}-\alpha u^{*}-P_{K}\left(v^{*}-\beta u^{*}+\gamma \epsilon^{*}\right),
$$

that is,

$$
v^{*}-\alpha u^{*}=P_{K}\left(v^{*}-\beta u^{*}+\gamma \epsilon^{*}\right) .
$$

Using (4.2), as in the proof of Theorem 3.1, we obtain that $\operatorname{SICP}(f, g, K)$ is feasible if $\gamma \geq 0$. Moreover, if $\gamma>0$, then $\operatorname{SICP}(f, g, K)$ is strictly feasible.

Case 2. Equation (4.1) does not have a solution, set $\psi=I-\frac{\beta}{\beta-\alpha} \phi$, then the mapping $\psi$ has no fixed point in $H$. For any $r>0$, let $U_{r}=\{x \in H:\|x\|<r\}$, the mapping $\psi$ is fixed-point free with respect to the set $\overline{U_{r}}$.

Since $f(x)=\frac{1}{\beta} x-S(x)$ and $g(x)=x-T(x)$, thus, for any $x \in H$, we have

$$
\psi(x)=\frac{\beta}{\beta-\alpha} T(x)-\frac{\alpha \beta}{\beta-\alpha} S(x)+P_{K}\left(\frac{\beta}{\beta-\alpha}(\beta S(x)-T(x)+\gamma \varepsilon(x))\right) .
$$

By the compactness of the mappings $S, T$ and $\varepsilon$, it is easy to see that $\psi$ is compact.

Since $I, P_{K}, f, g$ and $\varepsilon$ all are admissible, it follows from Lemma 2.1 that $\psi$ is admissible. Applying Theorem 2.1 with the restriction of the mapping $\psi$ and the set $U=U_{r}$, there exist $x_{r} \in \partial U_{r}=\{x \in H:\|x\|=r\}$ and $\lambda_{r}>1$ such that

$$
\lambda_{r} x_{r} \in \psi\left(x_{r}\right)=x_{r}-\frac{\beta}{\beta-\alpha} \phi\left(x_{r}\right) .
$$

Setting $\mu_{r}=\lambda_{r}-1$, it can be deduced from (4.3) that there exist $f^{r} \in f\left(x_{r}\right), g^{r} \in g\left(x_{r}\right)$ and $\varepsilon^{r} \in \varepsilon\left(x_{r}\right)$ such that

$$
\mu_{r} x_{r}+\frac{\beta}{\beta-\alpha} g^{r}-\frac{\alpha \beta}{\beta-\alpha} f^{r}=\frac{\beta}{\beta-\alpha} P_{K}\left(g^{r}-\beta f^{r}+\gamma \varepsilon^{r}\right) .
$$

Setting $s^{r}=\frac{\mu_{r}}{\beta} x_{r}+f^{r}-\frac{1}{\beta-\alpha} \gamma \varepsilon^{r}$ and $w^{r}=(\beta-\alpha) \mu_{r} x_{r}+\beta g^{r}-\alpha \beta f^{r}$, as in the proof of Theorem 3.1, we obtain that $\left\{x_{r}\right\}_{r>0}$ is an $(\alpha, \beta, \gamma)$-EFE for the pair $(f, g)$ with respect to $K$.

Remark 4.2 Since Definition 4.1 is a generalization of Definition 6.1 in [9], then Theorem 6.1 in [9] is a special case of Theorem 4.1.

Definition 4.2 Let $K$ be a closed pointed convex cone in a Hilbert space $H$ with int $K^{*} \neq \emptyset$. Let $f, g: H \rightarrow c(H)$ be two admissible mappings such that $f(x)=\frac{1}{\beta} x-S(x)$ and $g(x)=$ $\frac{1}{\beta} x-T(x)$, where $\beta>0, S, T: H \rightarrow c(H)$ are compact. Let $\varepsilon: H \rightarrow c\left(\right.$ int $\left.K^{*}\right)$ be a compact admissible mapping. Given $\alpha, \gamma \geq 0$, we say that the family of elements $\left\{x_{r}\right\}_{r>0} \subset H$ is an $(\alpha, \beta, \gamma)$-EFE for the pair $(f, g)$ with respect to $K$, if the following conditions are satisfied:

(1) $\left\|x_{r}\right\| \rightarrow+\infty$ as $r \rightarrow+\infty$;

(2) for any $r>0$, there exist $\mu_{r}>0$ and elements $f^{r} \in f\left(x_{r}\right), g^{r} \in g\left(x_{r}\right), \varepsilon^{r} \in \varepsilon\left(x_{r}\right)$ such that $s^{r}=\mu_{r} x_{r}+\beta f^{r}-\gamma \varepsilon^{r} \in K^{*}, w^{r}=\beta \mu_{r} x_{r}+\beta g^{r}-\alpha P_{K}\left(x_{r}-\beta f^{r}\right) \in K$ and $\left\langle s_{r}, w_{r}\right\rangle=0$. 


\section{Remark 4.3}

(1) If $g=I$ and $f$ is single-valued, Definition 4.2 reduces to Definition 3.2 in [11];

(2) If $g=I, f$ is single-valued and $\gamma=0$, Definition 4.2 reduces to Definition 3.2 in [8];

(3) If $g=I, f$ is single-valued and $\alpha=\gamma=0$, Definition 4.2 reduces to Definition 6.1 in [9].

Theorem 4.2 Let $H$ be a Hilbert space, and let $K \subset H$ be a closed convex cone with $\operatorname{int} K^{*} \neq \emptyset$. Let $\varepsilon: H \rightarrow c\left(\operatorname{int} K^{*}\right)$ be a compact admissible mapping, and let $f, g: H \rightarrow c(H)$ be two admissible mappings such that $f(x)=\frac{1}{\beta} x-S(x)$ and $g(x)=\frac{1}{\beta} x-T(x)$, where $\beta>0$, $S, T: H \rightarrow c(H)$ are compact. Then either $\operatorname{SICP}(f, g, K)$ is feasible, or for any $\alpha \geq 0$ and $\gamma \geq 0$, there exists an $(\alpha, \beta, \gamma)$-EFE (in the sense of Definition 4.2) for the pair $(f, g)$ with respect to $K$.

Moreover, if $\gamma>0$, then either $\operatorname{SICP}(f, g, K)$ is strictly feasible, or for any $\alpha \geq 0$, there exists an $(\alpha, \beta, \gamma)$-EFE (in the sense of Definition 4.2) for the pair $(f, g)$ with respect to $K$.

Proof Define $\phi: H \rightarrow c(H)$ by

$$
\begin{aligned}
\phi(x)= & \beta g(x)-\alpha P_{K}(x-\beta f(x)) \\
& -P_{K}\left(\beta g(x)-\beta f(x)+\gamma \varepsilon(x)-\alpha P_{K}(x-\beta f(x))\right), \quad \forall x \in H .
\end{aligned}
$$

Consider the equation

$$
x \in x-\phi(x) .
$$

We consider the following two cases.

Case 1. If equation (4.4) has a solution in $H$, denoted by $x^{*} \in H$, then there exist $u^{*} \in$ $\beta f\left(x^{*}\right), v^{*} \in \beta g\left(x^{*}\right)$ and $\epsilon^{*} \in \varepsilon\left(x^{*}\right)$ such that

$$
0=v^{*}-\alpha P_{K}\left(x^{*}-u^{*}\right)-P_{K}\left(v^{*}-u^{*}+\gamma \epsilon^{*}-\alpha P_{K}\left(x^{*}-u^{*}\right)\right),
$$

that is,

$$
v^{*}-\alpha P_{K}\left(x^{*}-u^{*}\right)=P_{K}\left(v^{*}-u^{*}+\gamma \epsilon^{*}-\alpha P_{K}\left(x^{*}-u^{*}\right)\right) .
$$

Using (4.5), as in the proof of Theorem 3.2, we obtain that the problem $\operatorname{SICP}(f, g, K)$ is feasible if $\gamma \geq 0$. Moreover, if $\gamma>0$, then $\operatorname{SICP}(f, g, K)$ is strictly feasible.

Case 2. If equation (4.4) does not have a solution, set $\psi=I-\phi$, then the mapping $\psi$ has no fixed point in $H$. From the representations of $f$ and $g$, we have

$$
\begin{aligned}
\psi(x)= & T(x)+\alpha P_{K}(S(x)) \\
& -P_{K}\left(S(x)-T(x)+\gamma \varepsilon(x)-\alpha P_{K}(S(x))\right), \quad \forall x \in H .
\end{aligned}
$$

For any $r>0$, let $U_{r}=\{x \in H:\|x\|<r\}$, then the mapping $\psi$ is fixed-point free with respect to the set $\overline{U_{r}}$.

By the compactness of the mappings $S, T$ and $\varepsilon$, it is clear that $\psi$ is compact.

Since $I, P_{K}, f, g$ and $\varepsilon$ all are admissible, it follows from Lemma 2.1 that $\psi$ is admissible. 
Applying Theorem 2.1 with the restriction of the mapping $\psi$ and the set $U=U_{r}$, we obtain that there exist $x_{r} \in \partial U_{r}=\{x \in H:\|x\|=r\}$ and $\lambda_{r}>1$ such that

$$
\begin{aligned}
\lambda_{r} x_{r} \in & x_{r}-\beta g\left(x_{r}\right)+\alpha P_{K}\left(x_{r}-\beta f\left(x_{r}\right)\right) \\
& \quad-P_{K}\left(g\left(x_{r}\right)-f\left(x_{r}\right)+\gamma \varepsilon\left(x_{r}\right)-\alpha P_{K}\left(x_{r}-\beta f\left(x_{r}\right)\right)\right) .
\end{aligned}
$$

Set $\mu_{r}=\frac{\lambda_{r}-1}{\beta}$. Then it follows from (4.6) that there exist $f^{r} \in f\left(x_{r}\right), g^{r} \in g\left(x_{r}\right)$ and $\varepsilon^{r} \in$ $\varepsilon\left(x_{r}\right)$ such that

$$
\beta \mu_{r} x_{r}+\beta g^{r}-\alpha P_{K}\left(x_{r}-\beta f^{r}\right)=P_{K}\left(\beta g^{r}-\beta f^{r}+\gamma \varepsilon^{r}-\alpha P_{K}\left(x_{r}-\beta f^{r}\right)\right) .
$$

Using (4.7), as in the proof of Theorem 3.2, we obtain that $\left\{x_{r}\right\}_{r>0}$ is an $(\alpha, \beta, \gamma)$-EFE for the pair $(f, g)$ with respect to $K$.

The following theorem presents a sufficient condition which ensures that the problem $\operatorname{SICP}(f, g, K)$ does not have an $(\alpha, \beta, \gamma)$-EFE for the pair $(f, g)$ with respect to $K$.

Theorem 4.3 Let $H$ be a Hilbert space, $K \subset H$ be a closed convex cone, and $f, g: H \rightarrow 2^{H}$ be two set-valued mappings satisfying the following condition.

(Condition $\left(\theta_{g}\right)$ ) If there exist $e \in K^{*}$ and a real number $\rho>0$ satisfying that for any $x \in K$ with $\|x\|>\rho$, there exists $x_{0} \in K$ such that for all $y \in f(x)$ and $z \in g(x)$, we have

$$
\left\langle z-x_{0}, y-e\right\rangle \geq 0 \text { and }\left\langle z-x_{0}, x\right\rangle>0
$$

Then $\operatorname{SICP}(f, g, K)$ does not have an $(\alpha, \beta, \gamma)$-EFE (in the sense of Definition 4.2) for the pair $(f, g)$ with respect to $K$. Thus, $\operatorname{SICP}(f, g, K)$ is strictly feasible.

Proof Define $\varepsilon: H \rightarrow c\left(\right.$ int $\left.K^{*}\right)$ by

$$
\varepsilon(x)=\{e\}, \quad \forall x \in H .
$$

We show that $\operatorname{SICP}(f, g, K)$ does not have an $(0,1,1)$-EFE (in the sense of Definition 4.2) for the pair $(f, g)$.

Suppose on the contrary that there exists an $(0,1,1)$-EFE $\left\{x_{r}\right\}_{r>0} \subset K$ for the pair $(f, g)$, that is, $\left\|x_{r}\right\| \rightarrow+\infty$ as $r \rightarrow+\infty$ and for any $r>0$, there exist $\mu_{r}>0, y_{r} \in f\left(x_{r}\right)$ and $z_{r} \in g\left(x_{r}\right)$ such that $s_{r}=\mu_{r} x_{r}+y_{r}-e \in K^{*}, w_{r}=\mu_{r} x_{r}+z_{r} \in K$ and $\left\langle s_{r}, w_{r}\right\rangle=0$.

For any $r>\rho,\left\|x_{r}\right\|=r$ implies that $\left\|x_{r}\right\|>\rho$, thus there exists an element $x_{0}^{r} \in K$ satisfying

$$
\left\langle z_{r}-x_{0}^{r}, y_{r}\right\rangle \geq 0 \text { and }\left\langle z_{r}-x_{0}^{r}, x_{r}\right\rangle>0
$$

Since $s_{r}=\mu_{r} x_{r}+y_{r}$, it can be deduced from (4.8) that $\left\langle z_{r}-x_{0}^{r}, s_{r}\right\rangle>0$.

Since $w_{r}=\mu_{r} x_{r}+z_{r}, x_{r}, x_{0}^{r} \in K$ and $s_{r} \in K^{*}$, it is obvious that

$$
0<\left\langle z_{r}-x_{0}^{r}, s_{r}\right\rangle=\left\langle w_{r}-\mu_{r} x_{r}-x_{0}^{r}, s_{r}\right\rangle=\left\langle w_{r}, s_{r}\right\rangle-\left\langle\mu_{r} x_{r}+x_{0}^{r}, s_{r}\right\rangle \leq 0,
$$

which is a contradiction.

Therefore, $\operatorname{SICP}(f, g, K)$ does not have an $(\alpha, \beta, \gamma)$-EFE for the pair $(f, g)$ with respect to $K$. Thus, by using Theorem $4.2, \operatorname{SICP}(f, g, K)$ is strictly feasible. 
Remark 4.4 The condition $\left(\theta_{g}\right)$ is a generalization of the Karamardian's condition. We refer the reader to $[5,18,19]$ for more details.

As a direct consequence of Theorem 4.3, we have the following corollary.

Corollary 4.1 Let $H$ be a Hilbert space, $K \subset H$ be a closed convex cone, and $f, g: H \rightarrow 2^{H}$ be two set-valued mappings satisfying the following condition.

(Condition $(\theta)$ ) If there exists $\rho>0$ such that for any $x \in K$ with $\|x\|>\rho$, there exists $x_{0} \in K$ such that for all $y \in f(x)$ and $z \in g(x)$, we have

$$
\left\langle z-x_{0}, y\right\rangle \geq 0 \quad \text { and } \quad\left\langle z-x_{0}, x\right\rangle>0
$$

\section{Then $\operatorname{SICP}(f, g, K)$ is strictly feasible.}

\section{Competing interests}

The authors declare that they have no competing interests.

\section{Authors' contributions}

All authors completed the paper together. All authors read and approved the final manuscript.

\section{Acknowledgements}

The author thanks the anonymous referees for their valuable remarks and suggestions, which helped to improve the article considerably. This work was supported by the National Natural Science Foundation of China (11061006), the National Natural Science Foundation of China (11226224), the Program for Excellent Talents in Guangxi Higher Education Institutions, the Guangxi Natural Science Foundation (2012GXNSFBA053008) and the Initial Scientific Research Foundation for PHD of Guangxi Normal University.

Received: 25 March 2013 Accepted: 20 May 2013 Published: 5 June 2013

\section{References}

1. Cottle, RW, Pang, JS, Stone, RE: The Linear Complementarity Problem. Academic Press, New York (1992)

2. Ferris, MC, Pang, JS: Engineering and economic applications of complementarity problems. SIAM Rev. 39, 669-713 (1997)

3. Bensoussan, A, Lions, J-L: Nouvelle formulation de problèmes de contròle impulsionnel et applications. C. R. Math. Acad. Sci. Paris, Sér. A-B 276, 1189-1192 (1973)

4. Harker, PT, Pang, JS: Finite-dimensional variational inequality and nonlinear complementarity problems: a survey of theory, algorithms and applications. Math. Program. 48, 161-220 (1990)

5. Karamardian, S: Complementarity problems over cones with monotone and pseudomonotone maps. J. Optim. Theory Appl. 18, 445-454 (1976)

6. Zhao, YB, Isac, G: Quasi- $P_{*}$-maps, $P(\tau, \alpha, \beta)$-maps, exceptional family of elements, and complementarity problems. J. Optim. Theory Appl. 105, 213-231 (2000)

7. Zhao, YB, Li, D: Strict feasibility conditions in nonlinear complementarity problems. J. Optim. Theory Appl. 107, 641-664 (2000)

8. Huang, N, Gao, C, Huang, X: Exceptional family of elements and feasibility for nonlinear complementarity problems. J. Glob. Optim. 25, 337-344 (2003)

9. Isac, G: Exceptional family of elements, feasibility and complementarity. J. Optim. Theory Appl. 104, $577-588$ (2000)

10. Isac, G, Kalashnikov, W: Exceptional families of elements, Leray-Schauder alternatives, pseudomonotone operators and complementarity. J. Optim. Theory Appl. 109, 69-83 (2001)

11. Yeol, JC, Li, J, Huang, N: Solvability of implicit complementarity problems. Math. Comput. Model. 45, 1001-1009 (2007)

12. Isac, G: On the implicit complementarity problem in Hilbert spaces. Bull. Aust. Math. Soc. 32, 251-260 (1985)

13. Isac, G: Fixed point theory and complementarity problems in Hilbert spaces. Bull. Aust. Math. Soc. 36, 295-310 (1987)

14. Górniewicz, L: Topological Fixed Point Theory of Multivalued Mappings. Kluwer Academic, Dordrecht (1999)

15. Isac, G, Bulavski, VA, Kalashnikov, W: Exceptional families, topological degree and complementarity problems. J. Glob. Optim. 10, 201-225 (1999)

16. Park, S: Generalized Leray-Schauder principles for condensing admissible multifunctions. Ann. Mat. Pura Appl. 172, 65-85 (1997)

17. In-Sook, K: Fixed point theorems for condensing admissible maps in topological vector spaces. Arch. Math. 80, 319-330 (2003)

18. Kalashnikov, W, Isac, G: Solvability of implicit complementarity problems. Ann. Oper. Res. 116, 199-221 (2002)

19. Karamardian, S: Generalized complementarity problem. J. Optim. Theory Appl. 8, 161-168 (1971) 
doi:10.1186/1029-242X-2013-284

Cite this article as: Zhong et al.: Feasibility of set-valued implicit complementarity problems. Journal of Inequalities and Applications 2013 2013:284.

Submit your manuscript to a SpringerOpen ${ }^{\circ}$ journal and benefit from:

- Convenient online submission

- Rigorous peer review

- Immediate publication on acceptance

Open access: articles freely available online

- High visibility within the field

- Retaining the copyright to your article

Submit your next manuscript at $\boldsymbol{s p r i n g e r o p e n . c o m ~}$ 